\title{
miRNAs: Important Targets for Oral Cancer Pain Research
}

\author{
Cláudia Maria Pereira, ${ }^{1,2}$ Dayany Sehnem, ${ }^{1}$ \\ Estevão Oliveira da Fonseca, ${ }^{3}$ Heráclito Fernando Gurgel Barboza, ${ }^{4}$ \\ Antônio Carlos Pires de Carvalho, ${ }^{5}$ Alexandre F. M. DaSilva, ${ }^{6}$ \\ Vivaldo Moura-Neto, ${ }^{1,7,8}$ and Marcos F. DosSantos ${ }^{7}$ \\ ${ }^{1}$ Programa de Pós-Graduação em Biomedicina Translacional, Universidade do Grande Rio (Unigranrio), Duque de Caxias, RJ, Brazil \\ ${ }^{2}$ Programa de Pós-Graduação em Odontologia Clínica e Experimental, Universidade do Grande Rio (Unigranrio), \\ Duque de Caxias, RJ, Brazil \\ ${ }^{3}$ Universidade Federal do Rio de Janeiro (UFRJ), Campus Macaé, Macaé, RJ, Brazil \\ ${ }^{4}$ Sociedade Brasileira para o Estudo da Dor (SBED), São Paulo, SP, Brazil \\ ${ }^{5}$ Programa de Pós-Graduação em Radiologia, Universidade Federal do Rio de Janeiro (UFRJ), Rio de Janeiro, RJ, Brazil \\ ${ }^{6}$ Headache \& Orofacial Pain Effort (HOPE), Department of Biologic and Materials Sciences \& Michigan Center for \\ Oral Health Research (MCOHR), School of Dentistry, University of Michigan, Ann Arbor, MI, USA \\ ${ }^{7}$ Instituto de Ciências Biomédicas (ICB), Universidade Federal do Rio de Janeiro (UFRJ), Rio de Janeiro, RJ, Brazil \\ ${ }^{8}$ Instituto Estadual do Cérebro Paulo Niemeyer (IECPN), Rio de Janeiro, RJ, Brazil
}

Correspondence should be addressed to Marcos F. DosSantos; santosmfh@gmail.com

Received 28 February 2017; Revised 28 August 2017; Accepted 14 September 2017; Published 30 October 2017

Academic Editor: Ming D. Li

Copyright (C) 2017 Cláudia Maria Pereira et al. This is an open access article distributed under the Creative Commons Attribution License, which permits unrestricted use, distribution, and reproduction in any medium, provided the original work is properly cited.

Pain is a symptom shared by an incredible number of diseases. It is also one of the primary conditions that prompt individuals to seek medical treatment. Head and neck squamous cell carcinoma (HNSCC) corresponds to a heterogeneous disease that may arise from many distinct structures of a large, highly complex, and intricate region. HNSCC affects a great number of patients worldwide and is directly associated with chronic pain, which is especially prominent during the advanced stages of oral squamous cell carcinoma (OSCC), an anatomical and clinical subtype that corresponds to the great majority oral cancers. Although the cellular and molecular bases of oral cancer pain have not been fully established yet, the results of recent studies suggest that different epigenetic mechanisms may contribute to this process. For instance, there is strong scientific evidence that microRNAs (miRNAs), small RNA molecules that do not encode proteins, might act by regulating the mechanisms underlying cancer-related pain. Among the miRNAs that could possibly interfere in pain-signaling pathways, miR-125b, miR-181, and miR-339 emerge as some of the most promising candidates. In fact, such molecules apparently contribute to inflammatory pain. Moreover, these molecules possibly influence the activity of endogenous pain control systems (e.g., opioidergic and serotonergic systems), which could ultimately result in peripheral and central sensitization, central nervous system (CNS) phenomena innately associated with chronic pain. This review paper focuses on the current scientific knowledge regarding the involvement of miRNAs in cancer pain, with special attention dedicated to OSCC-related pain.

\section{Background}

Head and neck squamous cell carcinoma (HNSCC), a prevalent and highly invasive cancer, is produced by unbalanced genetic and epigenetic events that take place in response to carcinogens. HNSCC affects a group of heterogeneous and intermingled structures, located in an incredibly complex region. HNSCC includes cancer in the oral cavity, larynx, and pharynx [1]. The main risk factors for HNSCC comprise behavior factors such as exposure to tobacco and alcohol abuse together with oncogenic viruses infections, especially human papilloma virus (HPV) [2]. Strikingly, less than 50\% 
of the patients that develop oral or pharyngeal cancer survive for more than five years, according to a late-stage diagnosis [3]. Considering the several subsets of malignant tumors in the head and neck, squamous cell carcinomas (SCCs) originated from the oral mucosa (OSCC) and oropharynx (OPSCC), ranked as the 14th most prevalent type of cancer among women and the eighth among men in the US [4]. OSCC accounts for approximately $80-90 \%$ of all malignancies of the oral cavity and preferentially affects the following anatomic sites: tongue, retromolar region, floor of the mouth, hard palate, and jugal mucosa [5]. Not surprisingly, roughly $75 \%$ of all OSCC cases are credited to tobacco exposure or alcohol abuse [6]. More recently, HPV infection has also been linked to a subset of OSCC [7] that exhibits a higher incidence in younger patients [4] and that is often associated with improved prognoses [8].

Notwithstanding substantial progress in the OSCC treatment has been witnessed in recent years, the effectiveness of the available therapies and recurrence are still relevant issues. Hence, there is a clear need for the discovery of new targets as well as signaling molecules in order to tailor more appropriate therapeutic strategies. Remarkably, it has been demonstrated that the overexpression of epidermal growth factor receptor (EGFR), a member of the ErbB receptor family, is associated with lower local tumor control after radiotherapy and a shortened overall survival in HNSCC $[9,10]$. Additionally, other markers have been investigated as possible immunotherapy targets for OSCC treatment [11]. For instance, the expression of cancer testis antigen (CTA) genes like MAGE A1 has been described in HNSCC [12] and in OSCC cells [11] but not in the normal oral mucosa cells (Figure 1).

HNSCC treatment is also challenging due to the considerable discrepancies in the therapeutic response among individuals. Therefore, the discovery and validation of novel biomarkers such as microRNAs (miRNAs) will contribute to more accurate treatment monitoring as well as the development of customized therapies, both with a direct impact on patients' quality of life [13]. In fact, changes in the circulating miRNAs have been found during the course of HNSCC radiochemotherapy, suggesting that some of those molecules (e.g., miR-425-5p and miR-93-5p) can be used as prognostic indicators, when evaluating the effects of cancer therapeutics [14]. Furthermore, according to a recent study, some polymorphisms in pre-miRNAs might influence the prognosis of squamous cell carcinomas of the nonoropharynx, particularly in patients undergoing chemoradiation and in smokers [15].

The presence of spontaneous pain prior to cancer therapy is also an important feature that must be considered when evaluating the prognosis of OSCC [16]. Nonetheless, despite the recent boom of research studies dedicated to find reliable biomarkers that might impact the response to the therapies and, therefore, the prognosis of OSCC [17-21], the number of studies that focus on the characterization of the molecular events related to oral cancer pain is still very limited. This fact might be explained by the relative low incidence of pain during the early stages of OSCC though its occurrence significantly increases over the course of the disease, becoming more pronounced at its later phases [22, 23]. According to some authors, the presence of pain in the early stages of OSCC depends on the anatomical site of the lesion, the activity of proinflammatory cytokines secreted by the invasive tumor cells, the presence of perineural/muscular invasion, and, finally, the existence of lymphoplasmacytic infiltration $[16,22,24]$.

\section{Pain in Cancer Patients}

Cancer-related pain represents a daunting challenge for both clinicians and pain researchers all over the globe. In fact, pain is among the concerning cancer symptoms, especially when considering the extremely negative impact that it exerts not only in the physical but also in the emotional state and cognitive functioning of the patients afflicted by such disease. Different subtypes of HNSCC have been connected to pain, which in many occasions can be excruciating and disabling, thus producing an tremendous deleterious influence over the quality of life and the functional activities of the individuals affected [25]. Overall, it has been estimated that around onethird of the patients that receive treatment for cancer and twothirds of those subjects who are in the advanced stages of the disease experience pain. Likewise, both the inflammatory and the neuropathic pain components may be present, contributing to its challenging clinical management [26].

A multiplicity of tumors (e.g., carcinomas and sarcomas) is associated with osteogenic metastasis and ends up producing spontaneous bone pain [11], a symptom that should receive considerable attention from clinicians. In fact, cancer patients that report pain throughout the clinical evaluation exhibit a considerable reduction of the pain threshold, explaining the presence of several pain phenomena routinely witnessed in those patients. For example, in some cases, even innocuous stimuli are capable of eliciting pain, a phenomenon defined as allodynia. In addition, an amplification of the painful response to harmful stimuli in a damaged tissue (primary hyperalgesia) or in the surrounding areas (secondary hyperalgesia) might be reported as well and strongly suggest that peripheral as well as central sensitization might take part in this process. These phenomena are often accompanied by a considerable increase in the levels of anxiety and depression [26, 27].

As previously described, although OSCC is usually asymptomatic during its initial stages, as in other types of cancer, pain might emerge during the subsequent phases. When present, functional pain remarkably surpasses spontaneous pain in OSCC patients [28]. Moreover, the presence of spontaneous pain before cancer treatment might predict an unfavorable prognosis of OSCC [16]. As a matter of fact, as previously reported, higher pain levels, which can be easily assessed at the clinical setting by the application of a visual analogue scale (VAS), predict the existence of perineural invasion in OSCC. Hence, a simple baseline pain evaluation allows clinicians to obtain a more accurate prognosis and to achieve an enhanced treatment decision in cancer patients [29].

Notwithstanding numerous past studies have confirmed the incidence of referred pain in cancer patients $[9,14]$, its 


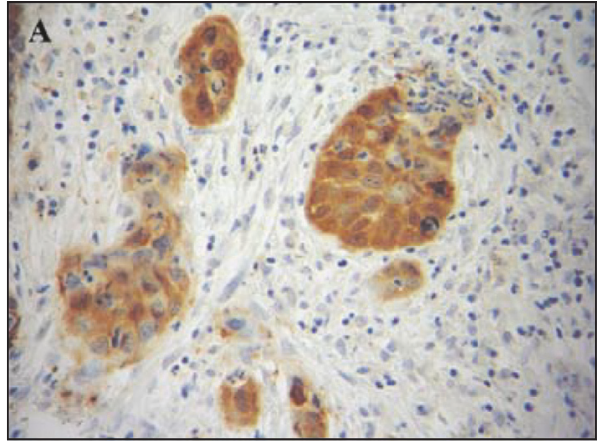

(a)

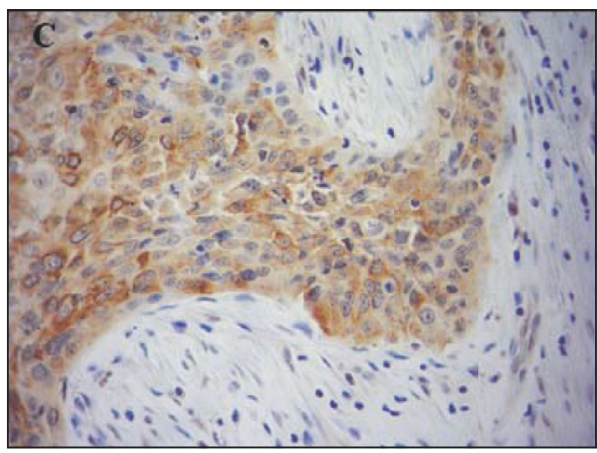

(c)

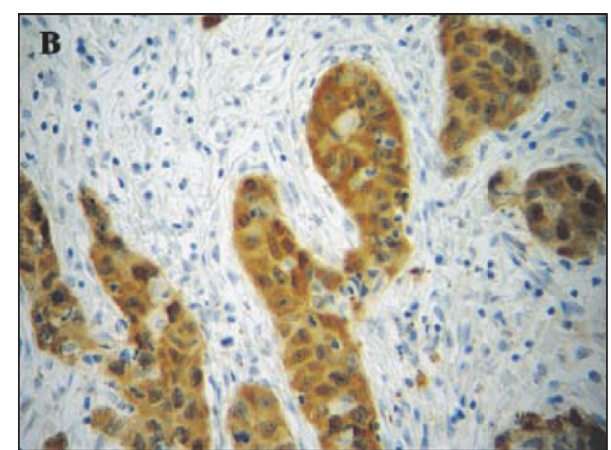

(b)

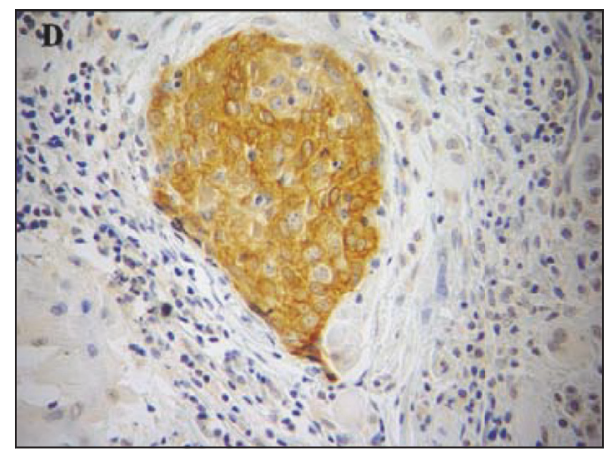

(d)

FIGURE 1: Immunohistochemical staining for MAGE A1 in oral squamous cell carcinoma (OSCC) samples. Cytoplasmic staining pattern in OSCC14 ( $\mathrm{a}$ and $\mathrm{b}$ ) and OSCC18 ( $\mathrm{c}$ and $\mathrm{d}$ ).

mechanisms remain largely unveiled. The elucidation of the cellular, biochemical, and molecular aspects of cancer pain is crucial to the identification of the events related to this phenomenon. Thus, future therapeutic strategies for OSCCrelated pain should rely not only on the signs and symptoms of the individuals affected but also on the pathophysiological mechanisms and dysfunctional signaling pathways implicated in the whole process. In this context, recent studies suggest that epigenetics (e.g., DNA methylation and miRNA) is a fundamental step in the deleterious transition from acute to chronic pain [30].

\section{How Are miRNAs Involved in Pain Mechanisms?}

miRNAs are a class of small RNAs (18-22 nucleotides) that do not encode proteins [31]. Nonetheless, those molecules do mediate gene expression through mechanisms of degradation or inhibition of the messenger RNA (mRNA) [32-34]. miRNAs can be actively exported from the intracellular to the extracellular space and this mechanism seems to be part of a complex response system that involves the participation of miRNAs in the cell-to-cell communication [35]. Slight fluctuations in the expression of signaling molecules, ion channels, and structural proteins, which in turn are directly linked to miRNAs activity, are inherently associated with the development and maintained of chronic pain [36].
A possible contribution of miRNAs to pain mechanisms has arisen few years ago, when a hypoexpression of miRNAs was demonstrated in the trigeminal ganglion neurons of an experimental model of inflammatory myogenic pain, clearly demonstrating that miRNAs can act at the peripheral nervous system and that changes in the expression of such key molecules can be related to the development of both allodynia and hyperalgesia [37].

In order to determine a signature of miRNAs associated with cancer pain, a previous study performed a wide genomewide screening of miRNAs in sensory neurons [26]. Among the set of miRNAs that exhibited altered expression, some miRNAs were validated as important modulators of tumorassociated hypersensitivity. Those findings support the investigation of miRNAs' contributions to chronic pain syndromes, including pain induced by cancer.

\section{Evidence of miRNAs Participation in Cancer-Related Pain?}

Despite the still scarce number of clinical studies, the scientific literature available suggests that epigenetic changes in the brain, as well as in the spinal cord, occur in different chronic pain conditions [27, 38-40]. Supporting this hypothesis, changes in the circulating miRNAs expressions have been previously demonstrated in different painful syndromes such as complex regional pain syndrome (CRPS) [41], fibromyalgia [42], 
irritable bowel syndrome [43], endometriosis [43], and osteoarthritis [44].

In irritable bowel syndrome (IBS), a disease with unclear mechanisms and difficult clinical management, the primary clinical visceral pain was correlated to a decreased expression of the colonic miR-199a/b in a previous study. Moreover, the upregulation of miR-199a reduced the IBS-related visceral pain [45]. In another study, an alternative pattern was demonstrated in migraine patients, wherein an acute upregulation of the miR-34a-5p and miR-382-5p expression was found during the migraine attacks [46]. Furthermore, miR-382-5p was overexpressed in migraine patients when compared to controls, not only during the headache attacks, but also during the pain-free periods. Interestingly, the same miR-382$5 \mathrm{p}$ is considered a brain-enriched miRNA, predominantly located in neurons and cerebrospinal fluid (CSF). Considering the possible involvement of a blood brain barrier (BBB) leakage in the migraine pathophysiology [47], it would be reasonable to consider that the increased concentrations of miR-382-5p observed in the serum could be derived from the CNS or the CSF [46]. Nevertheless, it is important to mention that though at lower concentrations, the miR-382-5p can be naturally detected in the serum [48].

Although the specific mechanisms whereby miRNAs contribute to nociception and pain are still not clear, there is mounting evidence that adaptive changes in the miRNAs expressions in response to tissue injury influence the activity of proinflammatory cytokines, which in turn mediate acute inflammatory pain $[27,49,50]$. Based on this concept, it is possible to hypothesize that a similar process underlies cancer pain.

In fact, it has been recognized that inflammatory mechanisms play a role in cancer establishment and progression. Those mechanisms potentially activate pain-signaling pathways. For example, a typical inflammatory response is characterized by an increased production of inflammatory mediators, including prostaglandin E2 (PGE2), interleukin 1 beta (IL-1 $\beta$ ), and tumor necrosis factor-alpha (TNF- $\alpha$ ) which also contribute to the development of hyperalgesia [51]. The same cytokines can affect the expression of miRNAs $[52,53]$ and might be modulated by miRNAs [54]. This hypothesis is sustained by the altered miRNAs regulation that occurs in metastatic bone areas, which was previously demonstrated in an experimental animal model of cancer pain. Moreover, the miRNAs targets found in the same study comprised painrelated genes [26].

All this growing evidence reinforces the promising use of miRNAs as potential targets in future pain therapies [55]. For instance, a previous work described that the increased inflammatory visceral pain in conjunction with the expression of the transient receptor vanilloid type 1 (TRPV1) was correlated to a decreased Gut miR-199a/b expression in IBS. Conversely, the upregulation of miR-199a reduced the IBS visceral pain through the inhibition of the TRPV1 signaling, indicating that miR-199a precursors might be clinically tested to the treatment of inflammatory visceral pain and in particular IBS [45].

Some promising miRNAs such as miR-125b-3p [56], miR125b-5p [42, 57], miR-181 [58], miR-30d-5p, miR-379-5p [57], and miR-339 [41] have been correlated to pain. Among the predicted targets for these miRNAs are some of the chief molecules for chronic pain, including the calcitonin gene related peptide (CGRP) $[59,60]$, TNF- $\alpha$ [61], transforming growth factor beta receptor 1 (TGFBR1) [62, 63], toll-like receptor (TLR-4) [64], interleukin 1 alfa (Il-1 $\alpha$ ) [65], interleukin 10 receptor, alpha subunit (IL-10RA) [66], and interleukin 6 receptor (IL-6R) [67]. Using genome-wide miRNA screening and molecular and in silico analyses, a previous study identified a subset of 57 miRNAs associated with metastatic bone-cancer pain in mice and validated miRNAs $1 a-3 p, 34 c-5 p$ 483-3p as important modulators of tumorassociated hypersensitivity [26]. A more recent study that applied in-depth bioinformatics analysis identified the Cav 2.3 as a target for miR-34c-5p [68]. Moreover, knockdown of Cav 2.3 in the dorsal root ganglion (DRG) of mice resulted in hypersensitivity, which clearly indicates an antinociceptive role of this calcium channel in sensory neurons.

Inflammation is also an important process related to the major cancer-related symptoms, including pain [69]. Not surprisingly, some miRNAs that are involved in the mechanisms of inflammation, including miR-181 family members as well as miR-125b, have targets that are also important to the nociceptive transmission. Indeed, a basic search using computerbased programs available for miRNA target prediction such as microRNA.org [70] and TargetScanHuman 7.1 [71] reveals that miR-181a-5p as well as mir-181b-5p might bind to the gamma-aminobutyric acid type A receptor, alpha 1 subunit (GABRA1). Supporting this information, an upregulation of miR-181a along with a downregulation of GABRA1 gene and protein expression in the dorsal horn of the spinal cord has been demonstrated in an experimental model of chronic inflammatory pelvic pain induced by neonatal cystitis [58]. Based on these results, it has been hypnotized that this downregulation of GABAA receptors induced by miR-181a could lead to a decreased inhibition in the spinal cord dorsal horn, contributing to a persistent visceral hypersensitivity [72].

The miR-181 family is composed by four members (miR181a, miR-181b, miR-181c, and miR-181d). These miRNAs are considered extremely important in the regulation of inflammation under physiological and pathological conditions [73]. In a previous study, miR-181a was able to inhibit the activity of the proinflammatory cytokine interleukin 8 (IL-8) of inflamed dental pulp tissue [74]. Thus, it is possible to speculate that this miRNA is directly involved in the regulation of pain and inflammation in the orofacial tissues. On the other hand, miR-125b-3p has been implicated in neuropathic as well as inflammatory pain, raising the possibility of contribution for cancer-related pain [59]. Variations of miR-125b$3 p$ expression have also been observed in the hippocampus of animals submitted to surgical chronic constriction of the sciatic nerve, a widely used experimental model of neuropathic pain [56]. Those results bring the intriguing question of whether a synaptic plasticity regulated by slight variations of miRNAs' expression in specific areas of the peripheral/ central nervous system could also be essential for neuropathic pain development.

Likewise, miR-339 seems to be important for cancerrelated pain. miR-339-3p is a natural target of mu-opioid 


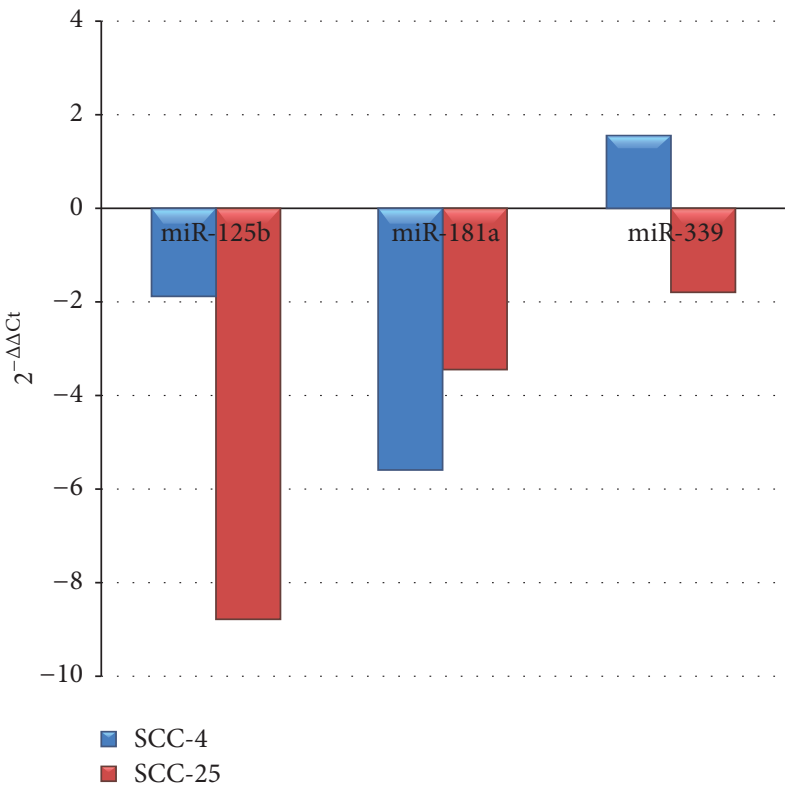

FIGURE 2: Expression of miR-125, miR-181, and miR-339 in OSCC cell lines. miRNAs expressions in SCC-4 (blue) and SCC-25 (red) compared to a immortalized keratinocyte cell line (HaCaT). These cell lines were obtained from the Rio de Janeiro Cell Bank (Banco de Células do Rio de Janeiro, BMCRJ) which originates from American Type Culture Collection (ATCC).

receptors. Those receptors are part of the opioidergic system, an important endogenous modulatory system and target of the great majority of the opioid drugs available for pain control. Those include morphine and related agonists, which are frequently used for cancer pain management [75-78].

An explorative analysis performed in OSCC cell lines reveals a marked change in the expressions of miR-125, miR-181, and miR-339 in OSCC cell lines, when compared to normal keratinocytes (Figure 2, unpublished data). This preliminary finding corroborates the participation of such molecules on the OSCC pathophysiology, demonstrated in previous studies. For instance, it has been reported that the overexpression of miR-181a and miR-181b may increase lymph-node metastasis, vascular invasion by tumor, and poor prognosis in OSCC patients, suggesting that this miRNA could be a potential biomarker of this disease [79]. Besides, downregulation of miR-125b has been found in OSCC cell lines and tumors and the transfection of the cells with exogenous miR-125b decreased cell proliferation and affected the expression of cancer-related genes [80]. Nonetheless, despite the scientific evidence supporting the involvement of these miRNAs in both neuropathic and inflammatory cancerrelated pain and their possible participation in the OSCC pathophysiology, it is still not possible to completely determine to which extent these findings are correlated to the clinical presentation and OSCC-induced pain. Therefore, further studies will be necessary to confirm this information and to expand the current knowledge regarding the miRNAs participation in chronic pain and particularly in cancerrelated pain. In this context, miR-199, miR-34a-5p, and miR$382-5 p$ are promising molecules that must be investigated in depth. In addition, other important topics in the field must be addressed in future works, such as the contribution of
miRNAs to the development of mucositis, a common effect of cancer therapy, that is usually characterized by excruciating pain, which in turn is often reported as the most prominent and disturbing symptom during the treatment for head and neck cancer [81, 82].

\section{Conclusions}

miRNAs have been considered potential biomarkers and possible therapeutic targets in a wide spectrum of clinical disorders, including chronic pain syndromes, with especial interest to cancer-related pain. Although numerous studies have improved the current understanding regarding the clinical manifestations of cancer-related pain, there remains unknowns concerning its pathophysiology as well as the disrupted signaling pathways involved. Therefore, the clarification of the cellular, biochemical, and molecular aspects related to cancer-induced pain is a fundamental step to the identification of the complex cascade of events that lead to this phenomenon. In the future, this knowledge will potentially allow the development of novel therapeutic strategies based on the dysfunctional mechanisms of cancer pain and not merely on the signs and symptoms reported. In this context, upcoming studies devoted to scrutinize the epigenetics of OSCC and other types of cancer, correlating such results to the clinical findings, will be crucial to evolve the clinical management of oral cancer-related pain.
Abbreviations
BBB: Blood brain barrier
CNS: Central nervous system
CGRP: Calcitonin gene related peptide 
CRPS: Complex regional pain syndrome

CSF: Cerebrospinal fluid

CTA: $\quad$ Cancer testis antigen

GABRA1: Gamma-aminobutyric acid type A receptor, alpha 1 subunit

HNSCC: Head and neck squamous cell carcinoma

IBS: Irritable bowel syndrome

Il-1 $\alpha: \quad$ Interleukin 1 alfa

IL-1 $\beta$ : Interleukin 1 beta

IL-6R: Interleukin 6 receptor

IL-8: $\quad$ Interleukin 8

IL-10RA: Interleukin 10 receptor

mRNA: Messenger RNA

miRNAs: microRNAs

OSCC: Oral squamous cell carcinoma

PGE2: Prostaglandin E2

TGFBR1: Transforming growth factor beta receptor 1

TLR-4: Toll-like receptor

TNF- $\alpha$ : Tumor necrosis factor-alpha

TRPV1: Transient receptor vanilloid type 1

VAS: Visual analogue scale.

SCC: $\quad$ Squamous cell carcinoma

OPSCC: Oropharyngeal squamous cell carcinoma.

\section{Conflicts of Interest}

The authors declare that they have no conflicts of interest.

\section{Authors' Contributions}

All authors designed the study, drafted, and reviewed the manuscript. Cláudia Maria Pereira and Dayany Sehnem conducted data analysis.

\section{Acknowledgments}

This study was supported by Coordenação de Aperfeiçoamento de Pessoal de Nível Superior (CAPES), Conselho Nacional de Desenvolvimento Científico e Tecnológico (CNPq), Brazil, and Fundação de Amparo à Pesquisa do Estado do Rio de Janeiro (FAPERJ).

\section{References}

[1] D. Tataru, V. Mak, R. Simo, E. A. Davies, and J. E. Gallagher, "Trends in the epidemiology of head and neck cancer in London," Clinical Otolaryngology, vol. 42, no. 1, pp. 104-114, 2017.

[2] S. Marur and A. A. Forastiere, "Head and Neck Squamous Cell Carcinoma: Update on Epidemiology, Diagnosis, and Treatment," Mayo Clinic Proceedings, vol. 91, no. 3, pp. 386-396, 2016.

[3] S. Fedele, "Diagnostic aids in the screening of oral cancer.," Head \& neck oncology, vol. 1, p. 5, 2009.

[4] M. L. Gillison, "Current topics in the epidemiology of oral cavity and oropharyngeal cancers," Head \& Neck, vol. 29, no. 8, pp. 779-792, 2007.
[5] B. K. Edwards, M. L. Brown, P. A. Wingo et al., "Annual report to the nation on the status of cancer, 1975-2002, featuring population-based trends in cancer treatment," Journal of the National Cancer Institute, vol. 97, no. 19, pp. 1407-1427, 2005.

[6] W. J. Blot, J. K. McLaughlin, D. M. Winn et al., "Smoking and drinking in relation to oral and pharyngeal cancer," Cancer Res, vol. 48, no. 11, pp. 3282-3287, 1988.

[7] A. K. Chaturvedi, E. A. Engels, W. F. Anderson, and M. L. Gillison, "Incidence trends for human papillomavirus-related and -unrelated oral squamous cell carcinomas in the United States," Journal of Clinical Oncology, vol. 26, no. 4, pp. 612-619, 2008.

[8] M. L. Gillison, W. M. Koch, R. B. Capone et al., "Evidence for a causal association between human papillomavirus and a subset of head and neck cancers," Journal of the National Cancer Institute, vol. 92, no. 9, pp. 709-720, 2000.

[9] M. Baumann and M. Krause, "Targeting the epidermal growth factor receptor in radiotherapy: Radiobiological mechanisms, preclinical and clinical results," Radiotherapy \& Oncology, vol. 72, no. 3, pp. 257-266, 2004.

[10] S. Keren, Z. Shoude, Z. Lu, and Y. Beibei, "Role of EGFR as a prognostic factor for survival in head and neck cancer: A metaanalysis," Tumor Biology, vol. 35, no. 3, pp. 2285-2295, 2014.

[11] C. M. Pereira, C. C. Gomes, J. D. F. C. Silva, M. B. Rodriguez, A. A. Barbosa, and R. S. Gomez, "Evaluation of MAGE A1 in oral squamous cell carcinoma," Oncology Reports, vol. 27, no. 6, pp. 1843-1848, 2012.

[12] F. T. Zamunér, B. T. R. Karia, C. Z. De Oliveira, C. R. Dos Santos, A. L. Carvalho, and A. L. Vettore, "A comprehensive expression analysis of cancer testis antigens in head and neck squamous cell carcinoma revels MAGEA3/6 as a marker for recurrence," Molecular Cancer Therapeutics, vol. 14, no. 3, pp. 828-834, 2015.

[13] R. Nagadia, P. Pandit, W. B. Coman, J. Cooper-White, and C. Punyadeera, "MiRNAs in head and neck cancer revisited," Cellular Oncology, vol. 36, no. 1, pp. 1-7, 2013.

[14] I. Summerer, M. Niyazi, K. Unger et al., "Changes in circulating microRNAs after radiochemotherapy in head and neck cancer patients," Journal of Radiation Oncology, vol. 8, no. 1, article 296, 2013.

[15] C. Wang, E. M. Sturgis, X. Chen, H. Zheng, Q. Wei, and G. $\mathrm{Li}$, "Pre-miRNA variants as predictors of clinical outcome in patients with squamous cell carcinomas of the nonoropharynx," Oncotarget, vol. 7, no. 18, pp. 26444-26453, 2016.

[16] J. Sato, Y. Yamazaki, A. Satoh et al., "Pain may predict poor prognosis in patients with oral squamous cell carcinoma," Oral Surgery, Oral Medicine, Oral Pathology, Oral Radiology, and Endodontology, vol. 111, no. 5, pp. 587-592, 2011.

[17] R. Werkmeister, B. Brandt, and U. Joos, "The erbB oncogenes as prognostic markers in oral squamous cell carcinomas," The American Journal of Surgery, vol. 172, no. 6, pp. 681-683, 1996.

[18] H. Myoung, M. J. Kim, J. H. Lee, Y. J. Ok, J. Y. Paeng, and P. Y. Yun, "Correlation of proliferative markers (Ki-67 and PCNA) with survival and lymph node metastasis in oral squamous cell carcinoma: a clinical and histopathological analysis of 113 patients," Int J Oral Maxillofac Surg, vol. 35, no. 11, pp. 1005-1010, 2006.

[19] L. S. Monteiro, M. Diniz-Freitas, T. Garcia-Caballero, S. Warnakulasuriya, J. Forteza, and M. Fraga, "Combined cytoplasmic and membranous EGFR and p53 overexpression is a poor prognostic marker in early stage oral squamous cell carcinoma," Journal of Oral Pathology \& Medicine, vol. 41, no. 7, pp. 559-567, 2012. 
[20] A. Min, C. Zhu, S. Peng, S. Rajthala, D. E. Costea, and D. Sapkota, "MicroRNAs as important players and biomarkers in oral carcinogenesis," BioMed Research International, vol. 2015, Article ID 186904, 2015.

[21] C. Rivera, A. K. Oliveira, R. A. P. Costa, T. De Rossi, and A. F. Paes Leme, "Prognostic biomarkers in oral squamous cell carcinoma: a systematic review," Oral Oncology, vol. 72, pp. 3847, 2017.

[22] J. Bagan, G. Sarrion, and Y. Jimenez, "Oral cancer: clinical features," Oral Oncology, vol. 46, no. 6, pp. 414-417, 2010.

[23] H.-B. Santos, T.-K. dos Santos, A.-R. Paz et al., "Clinical findings and risk factors to oral squamous cell carcinoma in young patients: A 12-year retrospective analysis," Medicina Oral Patología Oral y Cirugía Bucal, vol. 21, no. 2, pp. e151-e156, 2016.

[24] N. G. Nikitakis, E. Sarlani, A. Kolokythas et al., "Frequency of pain and correlation with clinical and histologic parameters in T1 squamous cell carcinoma of the tongue: a retrospective pilot study.", Journal of oral \& facial pain and headache, vol. 28, no. 1, pp. 46-51, 2014.

[25] B. L. Schmidt, "The Neurobiology of Cancer Pain," Journal of Oral and Maxillofacial Surgery, vol. 73, no. 12, pp. S132-S135, 2015.

[26] K. K. Bali, D. Selvaraj, V. P. Satagopam, J. Lu, R. Schneider, and R. Kuner, "Genome-wide identification and functional analyses of microRNA signatures associated with cancer pain," $E M B O$ Molecular Medicine, vol. 5, no. 11, pp. 1740-1758, 2013.

[27] G. Descalzi, D. Ikegami, T. Ushijima, E. J. Nestler, V. Zachariou, and M. Narita, "Epigenetic mechanisms of chronic pain," Trends in Neurosciences, vol. 38, no. 4, pp. 237-246, 2015.

[28] S. T. Connelly and B. L. Schmidt, "Evaluation of pain in patients with oral squamous cell carcinoma," The Journal of Pain, vol. 5, no. 9, pp. 505-510, 2004.

[29] C.-F. Yeh, W.-Y. Li, P.-Y. Chu et al., "Pretreatment pain predicts perineural invasion in oral squamous cell carcinoma: A prospective study," Oral Oncology, vol. 61, pp. 115-119, 2016.

[30] T. Buchheit, T. Van de Ven, and A. Shaw, "Epigenetics and the Transition from Acute to Chronic Pain," Pain Medicine, vol. 13, no. 11, pp. 1474-1490, 2012.

[31] M. V. Iorio and C. M. Croce, "MicroRNAs in cancer: small molecules with a huge impact," Journal of Clinical Oncology, vol. 27, no. 34, pp. 5848-5856, 2009.

[32] D. P. Bartel, "MicroRNAs: genomics, biogenesis, mechanism, and function," Cell, vol. 116, no. 2, pp. 281-297, 2004.

[33] A. L. Gartel and E. S. Kandel, "RNA interference in cancer," Biomolecular Engineering, vol. 23, no. 1, pp. 17-34, 2006.

[34] Z. Zhang, Y.-Q. Cai, F. Zou, B. Bie, and Z. Z. Pan, "Epigenetic suppression of GAD65 expression mediates persistent pain," Nature Medicine, vol. 17, no. 11, pp. 1448-1455, 2011.

[35] K. Wang, S. Zhang, J. Weber, D. Baxter, and D. J. Galas, "Export of microRNAs and microRNA-protective protein by mammalian cells," Nucleic Acids Research, vol. 38, no. 20, pp. 7248-7259, 2010.

[36] E. Niederberger, K. Kynast, J. Lötsch, and G. Geisslinger, "MicroRNAs as new players in the pain game," PAIN, vol. 152, no. 7, pp. 1455-1458, 2011.

[37] G. Bai, R. Ambalavanar, D. Wei, and D. Dessem, "Downregulation of selective microRNAs in trigeminal ganglion neurons following inflammatory muscle pain," Molecular Pain, vol. 3, article no. 15, 2007.

[38] M. Tajerian, S. Alvarado, M. Millecamps et al., "Peripheral nerve injury is associated with chronic, reversible changes in global
DNA methylation in the mouse prefrontal cortex," PLoS ONE, vol. 8, no. 1, Article ID e55259, 2013.

[39] S. Imai, M. Saeki, M. Yanase et al., "Change in microRNAS associated with neuronal adaptive responses in the nucleus accumbens under neuropathic pain," The Journal of Neuroscience, vol. 31, no. 43, pp. 15294-15299, 2011.

[40] M. Kress, A. Hüttenhofer, M. Landry et al., "microRNAs in nociceptive circuits as predictors of future clinical applications," Frontiers in Molecular Neuroscience, vol. 6, 2013.

[41] I. A. Orlova, G. M. Alexander, R. A. Qureshi et al., "MicroRNA modulation in complex regional pain syndrome," Journal of Translational Medicine, vol. 9, no. 1, article no. 195, 2011.

[42] J. L. Bjersing, C. Lundborg, M. I. Bokarewa, and K. Mannerkorpi, "Profile of Cerebrospinal microRNAs in Fibromyalgia," PLoS ONE, vol. 8, no. 10, Article ID e78762, 2013.

[43] Q. Zhou, W. W. Souba, C. M. Croce, and G. N. Verne, "MicroRNA-29a regulates intestinal membrane permeability in patients with irritable bowel syndrome," Gut, vol. 59, no. 6, pp. 775-784, 2010

[44] C. Beyer, A. Zampetaki, N. Lin et al., "Signature of circulating microRNAs in osteoarthritis," Annals of the Rheumatic Diseases, vol. 74, no. 3, pp. e18-e18, 2015.

[45] Q. Zhou, L. Yang, S. Larson et al., "Decreased miR-199 augments visceral pain in patients with IBS through translational upregulation of TRPV1," Gut, vol. 65, no. 5, pp. 797-805, 2016.

[46] H. H. Andersen, M. Duroux, and P. Gazerani, "Serum MicroRNA Signatures in Migraineurs During Attacks and in Pain-Free Periods," Molecular Neurobiology, vol. 53, no. 3, pp. 1494-1500, 2016.

[47] M. F. DosSantos, R. C. Holanda-Afonso, R. L. Lima, A. F. DaSilva, and V. Moura-Neto, "The role of the blood-brain barrier in the development and treatment of migraine and other pain disorders," Frontiers in Cellular Neuroscience, vol. 8, article no. 302, 2014.

[48] K. L. Burgos, A. Javaherian, R. Bomprezzi et al., "Identification of extracellular miRNA in human cerebrospinal fluid by nextgeneration sequencing," RNA, vol. 19, no. 5, pp. 712-722, 2013.

[49] A. Sakai and H. Suzuki, "Nerve injury-induced upregulation of miR-21 in the primary sensory neurons contributes to neuropathic pain in rats," Biochemical and Biophysical Research Communications, vol. 435, no. 2, pp. 176-181, 2013.

[50] C. I. Svensson, "Interleukin-6: A local pain trigger?" Arthritis Research \& Therapy, vol. 12, no. 5, article no. 145, 2010.

[51] V. Gangadharan and R. Kuner, "Pain hypersensitivity mechanisms at a glance," Disease Models \& Mechanisms, vol. 6, no. 4, pp. 889-895, 2013.

[52] V. Benes, P. Collier, C. Kordes et al., "Identification of cytokineinduced modulation of microRNA expression and secretion as measured by a novel microRNA specific qPCR assay," Scientific Reports, vol. 5, Article ID 11590, 2015.

[53] J. Grace and B. Hall, "Comparison of flow cytometric data obtained using fresh and paraffin-embedded lymphoid tissue," Anal Quant Cytol Histol, vol. 11, no. 1, pp. 67-71, 1989.

[54] J.-S. Kim, S.-K. Yu, M.-H. Lee et al., "MicroRNA-205 directly regulates the tumor suppressor, interleukin-24, in human $\mathrm{KB}$ oral cancer cells," Molecules and Cells, vol. 35, no. 1, pp. 17-24, 2013.

[55] A. J. Asirvatham, W. J. Magner, and T. B. Tomasi, "miRNA regulation of cytokine genes," Cytokine, vol. 45, no. 2, pp. 5869, 2009. 
[56] M. Arai, Y. Genda, M. Ishikawa, T. Shunsuke, T. Okabe, and A. Sakamoto, "The miRNA and mRNA Changes in Rat Hippocampi after Chronic Constriction Injury," Pain Medicine, vol. 14, no. 5, pp. 720-729, 2013.

[57] K. K. Bali, M. Hackenberg, A. Lubin, R. Kuner, and M. Devor, "Sources of individual variability: MiRNAs that predispose to neuropathic pain identified using genome-wide sequencing," Molecular Pain, vol. 10, no. 1, article no. 22, 2014.

[58] J. N. Sengupta, S. Pochiraju, P. Kannampalli et al., "Erratum: MicroRNA-mediated GABAA $\alpha$-1 receptor subunit downregulation in adult spinal cord following neonatal cystitisinduced chronic visceral pain in rats (Pain (2012) 154:1 (59-70))," PAIN, vol. 154, no. 3, p. 493, 2013.

[59] Y. Dong, P. Li, Y. Ni, J. Zhao, and Z. Liu, "Decreased microRNA$125 \mathrm{a}-3 \mathrm{p}$ contributes to upregulation of p38 MAPK in rat trigeminal ganglions with orofacial inflammatory pain," PLoS ONE, vol. 9, no. 11, Article ID el11594, 2014.

[60] F. Zhang, X. Fan, Y. Bai et al., "MiR-125b regulates procalcitonin production in monocytes by targeting Stat3," Microbes and Infection, vol. 18, no. 2, pp. 102-108, 2016.

[61] C. Dan, B. Jinjun, H. Zi-Chun et al., "Modulation of TNF- $\alpha$ mRNA stability by human antigen $\mathrm{R}$ and miR181s in sepsisinduced immunoparalysis," EMBO Molecular Medicine, vol. 7, no. 2, pp. 140-157, 2015.

[62] L. Liu, Y. Wang, H. Fan et al., "MicroRNA-181a regulates local immune balance by inhibiting proliferation and immunosuppressive properties of mesenchymal stem cells," Stem Cells, vol. 30, no. 8, pp. 1756-1770, 2012.

[63] X. He, Z. Liu, Y. Peng, and C. Yu, "MicroRNA-181c inhibits glioblastoma cell invasion, migration and mesenchymal transition by targeting TGF- $\beta$ pathway," Biochemical and Biophysical Research Communications, vol. 469, no. 4, pp. 1041-1048, 2016.

[64] C. Feng, M. Bai, N.-Z. Yu, X.-J. Wang, and Z. Liu, "MicroRNA$181 \mathrm{~b}$ negatively regulates the proliferation of human epidermal keratinocytes in psoriasis through targeting TLR4," Journal of Cellular and Molecular Medicine, vol. 21, no. 2, pp. 278-285, 2017.

[65] X. Jiang, C. Xu, F. Lei et al., "MiR-30a targets IL-1 $\alpha$ and regulates islet functions as an inflammation buffer and response factor," Scientific Reports, vol. 7, no. 1, 2017.

[66] A.-L. Gagez, I. Duroux-Richard, S. Leprêtre et al., "MIR-125b and miR-532-3p predict the efficiency of rituximab-mediated lymphodepletion in chronic lymphocytic leukemia patients. A French Innovative Leukemia Organization study," Haematologica, vol. 102, no. 4, pp. 746-754, 2017.

[67] J. Gong, J.-P. Zhang, B. Li et al., "MicroRNA-125b promotes apoptosis by regulating the expression of Mcl-1, Bcl-w and IL6R," Oncogene, vol. 32, no. 25, pp. 3071-3079, 2013.

[68] J. Gandla, S. K. Lomada, J. Lu, R. Kuner, and K. K. Bali, "miR$34 c-5 p$ functions as pronociceptive microRNA in cancer pain by targeting Cav2.3 containing calcium channels," PAIN, vol. 158, no. 9, pp. 1765-1779, 2017.

[69] B. J. Laird, D. C. McMillan, P. Fayers et al., "The systemic inflammatory response and its relationship to pain and other symptoms in advanced cancer," The Oncologist, vol. 18, no. 9, pp. 1050-1055, 2013.

[70] D. Betel, M. Wilson, A. Gabow, D. S. Marks, and C. Sander, “The microRNA.org resource: targets and expression," Nucleic Acids Research, vol. 36, no. 1, pp. D149-D153, 2008.

[71] X. Song, L. Cheng, T. Zhou et al., "Predicting miRNA-mediated gene silencing mode based on miRNA-target duplex features," Computers in Biology and Medicine, vol. 42, no. 1, pp. 1-7, 2012.
[72] M. J. López-González, M. Landry, and A. Favereaux, "MicroRNA and chronic pain: From mechanisms to therapeutic potential," Pharmacology \& Therapeutics, 2017.

[73] X. Sun, A. Sit, and M. W. Feinberg, "Role of miR-181 family in regulating vascular inflammation and immunity," Trends in Cardiovascular Medicine, vol. 24, no. 3, pp. 105-112, 2014.

[74] J. C. Galicia, A. R. Naqvi, C.-C. Ko, S. Nares, and A. A. Khan, "MiRNA-181a regulates Toll-like receptor agonist-induced inflammatory response in human fibroblasts," Genes \& Immunity, vol. 15, no. 5, pp. 333-337, 2014.

[75] Q. Wu, C. K. Hwang, H. Zheng et al., "MicroRNA 339 downregulates $\mu$-opioid $\mu$ eceptor at the post-transcriptional level in response to opioid treatment," The FASEB Journal, vol. 27, no. 2 , pp. 522-535, 2013.

[76] N. Marie, B. Aguila, and S. Allouche, "Tracking the opioid receptors on the way of desensitization," Cellular Signalling, vol. 18, no. 11, pp. 1815-1833, 2006.

[77] S. B. McMahon, Wall and Melzack's Textbook of Pain, Elsevier/Saunders. xxix, Philadelphia, PA, USA, 6th edition, 2013.

[78] M. J. Brownstein, "A brief history of opiates, opioid peptides, and opioid receptors." Proceedings of the National Acadamy of Sciences of the United States of America, vol. 90, no. 12, pp. 53915393, 1993.

[79] C.-C. Yang, P.-S. Hung, P.-W. Wang et al., "miR-181 as a putative biomarker for lymph-node metastasis of oral squamous cell carcinoma," Journal of Oral Pathology \& Medicine, vol. 40, no. 5, pp. 397-404, 2011.

[80] B. J. Henson, S. Bhattacharjee, D. M. O’Dee, E. Feingold, and S. M. Gollin, "Decreased expression of miR-125b and miR100 in oral cancer cells contributes to malignancy," Genes, Chromosomes and Cancer, vol. 48, no. 7, pp. 569-582, 2009.

[81] A. M. Rose-Ped, L. A. Bellm, J. B. Epstein, A. Trotti, C. Gwede, and H. J. Fuchs, "Complications of radiation therapy for head and neck cancers: The patient's perspective," Cancer Nursing, vol. 25, no. 6, pp. 461-469, 2002.

[82] D. J. Harris, "Cancer treatment-induced mucositis pain: Strategies for assessment and management," Therapeutics and Clinical Risk Management, vol. 2, no. 3, pp. 251-258, 2006. 


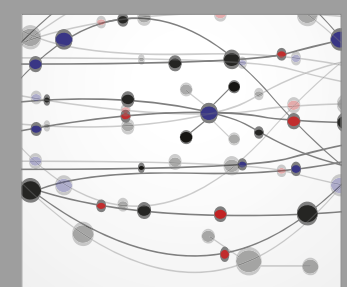

The Scientific World Journal
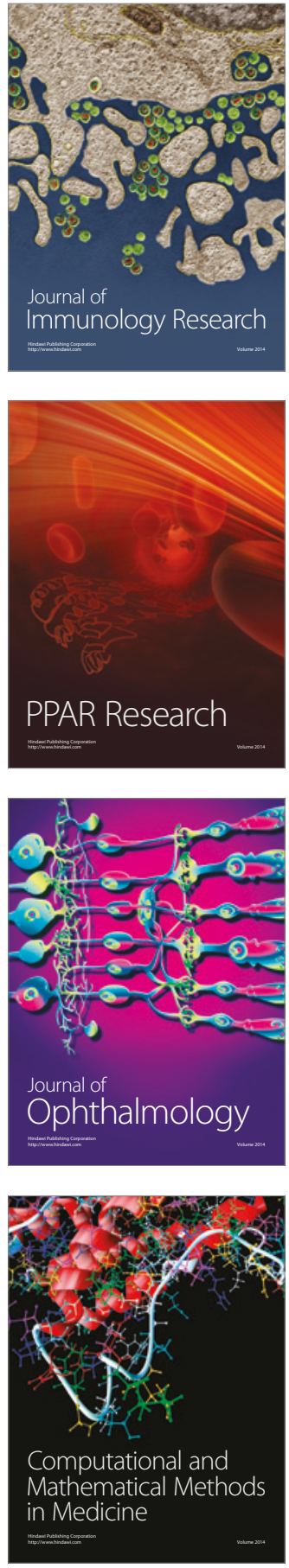

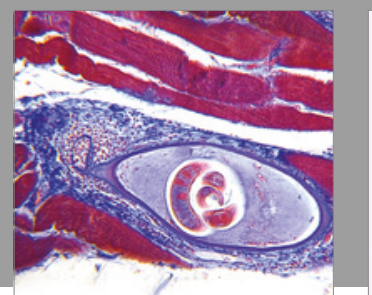

Gastroenterology Research and Practice
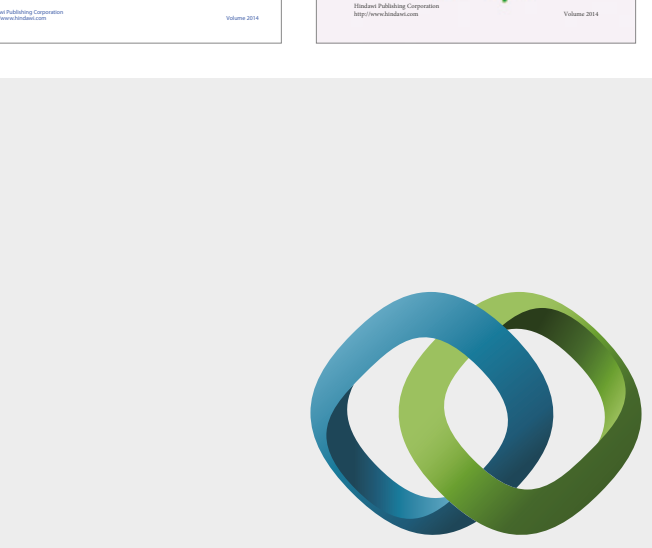

\section{Hindawi}

Submit your manuscripts at

https://www.hindawi.com
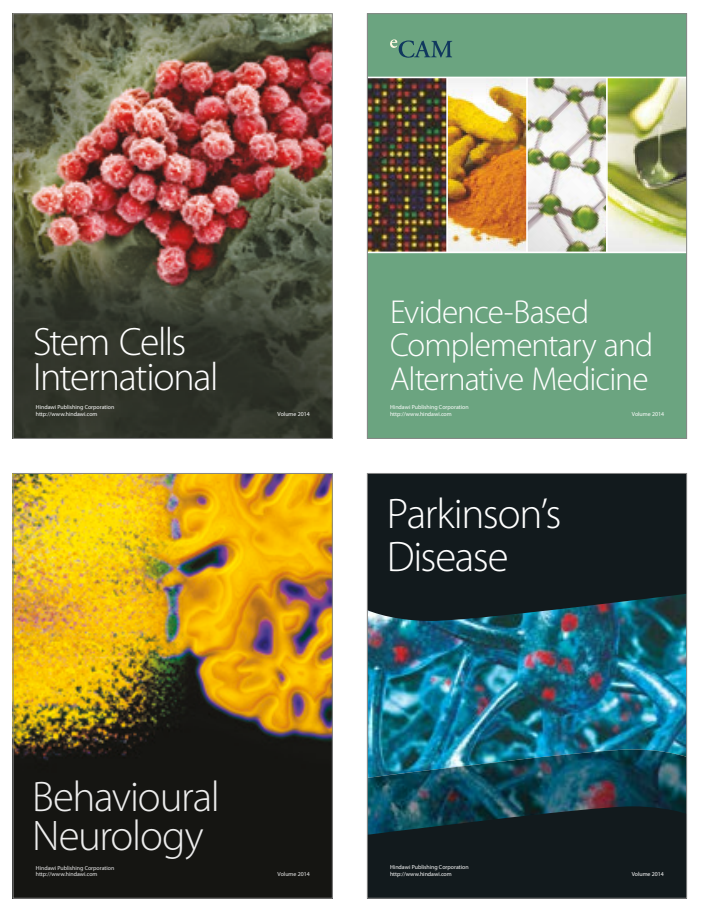
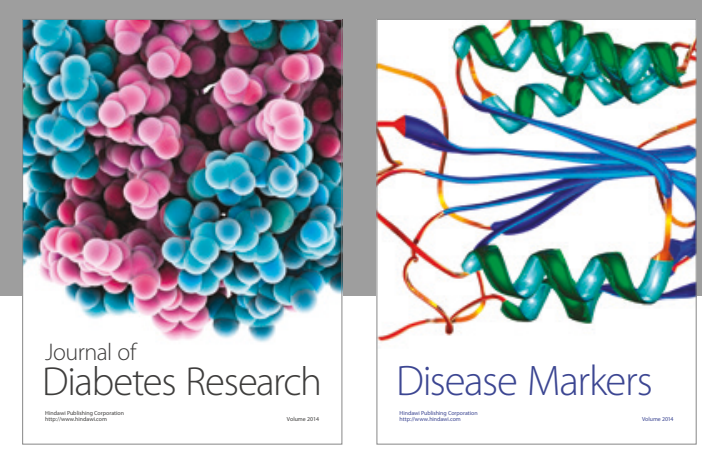

Disease Markers
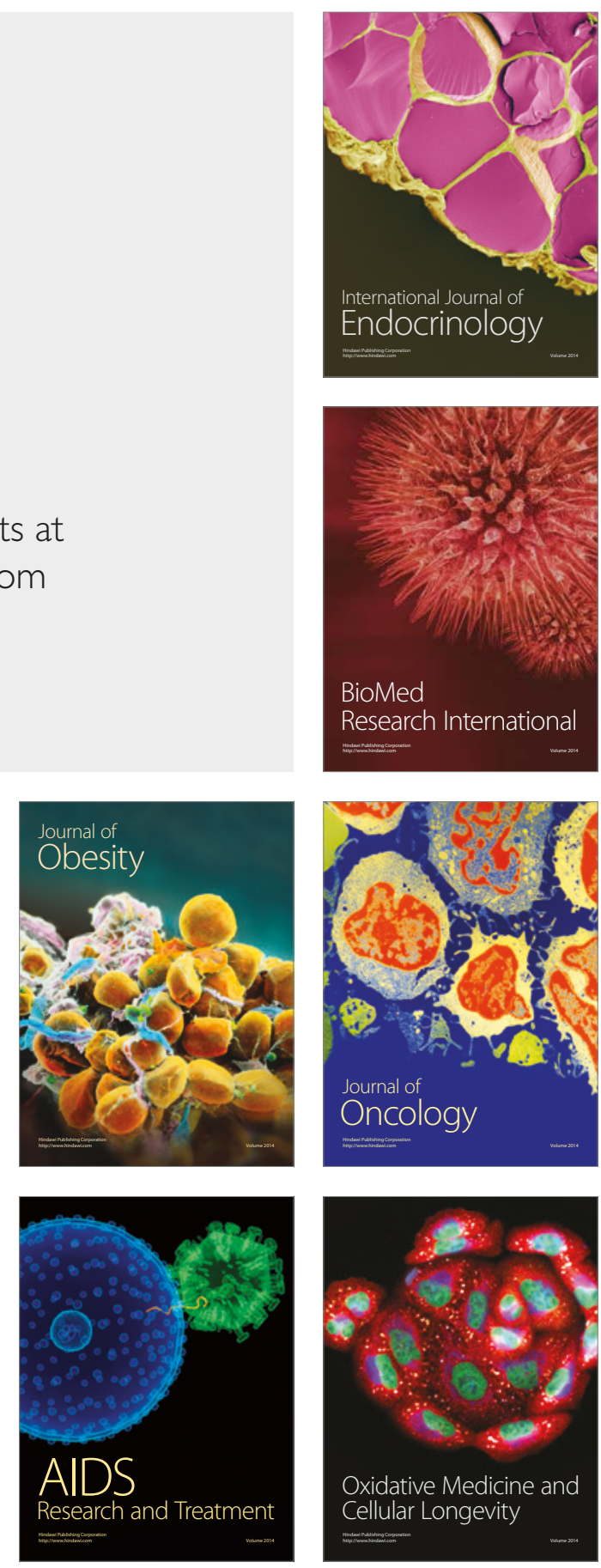